\title{
Spectrophone Measurements in Sulfur Hexafluoride
}

\author{
MANAF H. ALI, HENRY E. BASS, AND HAI-XING YAN
}

\begin{abstract}
The optoacoustic signal generated by pulsed $10.6 \mu$ infrared radiation incident upon a test cell filled with gaseous $\mathbf{S F}_{6}$ has been analyzed in detail. The effects of microscopic energy transfer from the absorbing vibrational degrees of freedom, spontaneous emission, thermal conduction, and acoustic wave propagation are included. This complete Ireatment explains the experimental observations including a negative pressure response following irradiation at low gas pressure.
\end{abstract}

\section{INTRODUCTION}

A LEXANDER BELL observed in 1880 [1] that sound can be produced by exposing a gas in a constant-volume container to intensity-modulated infrared radiation. The system used to observe this effect is the spectrophone. The process of converting optical energy to sound, referred to as the optoacoustic effect, can be explained in terms of molecular theory [2]. When molecules absorb infrared radiation, the energy appears as quanta of vibrational energy. A molecule may lose this energy by spontaneous emission of radiation, by induced emission, or by transfer of energy to thermal energy of motion. This last process occurs in collisions when vibrational energy is transferred to translation. An increase in translational energy means the gas will be heated and reach a new temperature. If the system is illuminated by infrared radiation that is periodically interrupted, the gas will heat and cool, producing a periodic change of pressure in a system of constant volume. These periodic pressure changes can be detected with a microphone.

Interpretation of spectrophone data requires accurate relations between the microscopic energy transfer processes and the measured pressure variation as well as a description of other macroscopic processes that affect the signal, e.g., acoustic propagation and thermal conduction. Bauer [3] has developed a general theory for relating the microscopic energy transfer processes to the optoacoustic response for a gas with multiple internal energy levels. Bass and Yan [4] have modified Bauer's theory to include thermal conduction and wall collisions.

In this work energy transfer in $\mathrm{SF}_{6}$ has been explored

Manuscript received December 14, 1985; revised March 17, 1986. This work was supported by the Office of Naval Research.

M. H. Ali is with Al-Dawoodi, 17/46 Hay Al-Koudat, Baghdad, Iraq He was with the Physical Acoustics Research Laboratory, The University of Mississippi, University, MI 38677, USA.

H. E. Bass is with the Physical Acoustics Research Laboratory, The University of Mississippi, University, MI 38677. USA.

H. X. Yan is with the Division of Laser Research, Institute of Mechanics, Chinese Academy of Sciences, Beijing, People's Republic of China. He was with the Physical Acoustics Research Laboratory, The University of Mississippi, University, MI 38677, USA

IEEE Log Number 8609272.

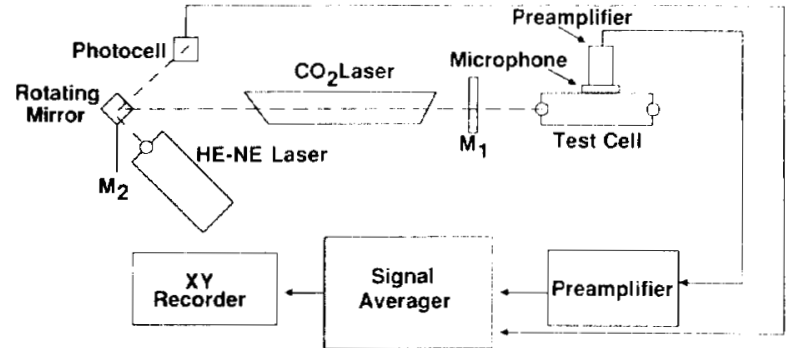

Fig. 1. Block diagram of the experimental apparatus.

using a pulsed spectrophone. The $\mathrm{SF}_{6}$ molecular system was chosen because energy transfer in $\mathrm{SF}_{6}$ has been thoroughly studied using other techniques [2], [5], and it strongly absorbs $\mathrm{CO}_{2}$ laser radiation.

\section{DESCRIPTION OF THE EXPERIMENT}

The experimental apparatus consists of a source of pulsed IR radiation, the test cell, a microphone, gas handling equipment, and signal-averaging electronics as shown in Fig. 1. A detailed description of the experimental technique has been previously published [4].

$\mathrm{SF}_{6}$ in a cylindrical test cell was illuminated with a short burst approximately $1 \mu \mathrm{s}$ in duration from a $Q$-switched $\mathrm{CO}_{2}$ laser. The test cell has IR windows on both ends to allow the laser beam to enter and exit. The acoustic signal was picked up by a microphone mounted in the cell wall near the entrance window. After the signal had passed through the preamplifier and signal averager, it was recorded on an $x-y$ recorder.

\section{THEORY}

The equation for heat flow in the test cell with internal sources of heat can be written as [6]

$$
\frac{\partial \theta(r, t)}{\partial t}=\kappa\left(\frac{\partial^{2} \theta(r, t)}{\partial r^{2}}+\frac{1}{r} \frac{\partial \theta(r, t)}{\partial r}\right)+H(r, t)
$$

where $\theta$ is the difference between the gas and tube temperatures (the tube temperature is assumed to be constant). Both $\theta$ and $H$ are also functions of $z$ (distance down the test cell). This dependence has been separated out and will be treated separately later. Separation of variables at this point differs from the approach used in [7], where a time-dependent eigenvalue analysis was employed.

The rate of heat generation is assumed to be a product of space and time-dependent parts

$$
H(r, t)=\frac{\kappa}{k} f(r) g(t)
$$


where $\mathrm{k}$ is the thermal diffusivity and $k$ is the thermal conductivity $\left(k={ }_{k} C_{v} P\right.$ ). The function $g(t)$ is the heat generated by relaxation processes which is equal to $\partial u / \partial t$, where $u$ is the energy flow into translation.

Prior to proceeding further with the solution of (1), a form for $f(r)$ must be assumed. We will assume a uniform radial excitation though some type of a Gaussian distribution across the cell radius might be more appropriate.

For uniform excitation, $f(r)$ is equal to one so that $H(r$, $t)$ is equal to $(\kappa / k)(\partial u(t) / \partial t)$. Equation (1) can then be written as

$$
\frac{\partial}{\partial r}\left[\theta(r, t)-\frac{\kappa}{k} u(t)\right]=\kappa\left(\frac{\partial^{2} \theta(r, t)}{\partial r^{2}}+\frac{1}{r} \frac{\partial \theta(r, t)}{\partial r}\right)
$$

Equation (3) can be solved by separation of variables. Set $\theta(r, t)$ equal to $R(r) Q(t)$. Then by substituting $\theta(r, t)$ into Equation (3) and dividing by $R(r) Q(t)$, the equation for heat flow becomes

$$
\frac{1}{\kappa R Q(t)} \frac{\partial}{\partial t}\left[Q(t)-\frac{\kappa}{k} u\right]=\frac{1}{R(r)}\left[\frac{\partial^{2}}{\partial r^{2}} R(r)\right]+\frac{1}{r} \frac{\partial}{\partial r} R(r)
$$

By equating (4) to $-\alpha^{2}$, the radial equation (right-hand side), has the solution $R=A J_{0}(\alpha r)$, where $J_{0}$ is Bessel's function and $A$ is a normalization constant to be determined from the boundary conditions.

The boundary conditions require that $\theta=0$ when $r=$ the cell radius $a$ hence $J_{0}\left(\alpha_{m} a\right)=0$ and $\alpha_{1}, \alpha_{2}, \cdots, \alpha_{m}$ are zeros of the Bessel function. The radial solution is then

$$
R(r)=\sum_{m} A_{m} J_{0}\left(\alpha_{m} r\right) .
$$

$A_{m}$ can be determined to be $2 / a \alpha_{m} J_{1}\left(\alpha_{m} a\right)$ so

$$
\theta(r, t)=2 \sum_{m} \frac{J_{0}\left(\alpha_{m} r\right)}{a \alpha_{m} J_{1}\left(\alpha_{m} a\right)} Q(t)
$$

Next, (6) is averaged across the radius of the test cell giving

$$
\theta(t)=\frac{2}{a^{2}} \int_{0}^{a} \theta(r, t) d r=4 Q(t) \sum_{m} \frac{1}{a^{2} \alpha_{m}^{2}}
$$

where

$$
Q(t)=\frac{1}{4} \theta(t)\left[\sum_{m} \frac{1}{a^{2} \alpha_{m}^{2}}\right]^{-1} .
$$

The justification for averaging over $r$ is based upon the lack of any measurable radial oscillations, which would exhibit themselves as high frequency variations on the measured pressure waveform. Equation (4) can now be written as

$$
\frac{\partial}{\partial t}\left[Q(t) \sum_{m} R_{m}(r)-\frac{\kappa}{k} u(t)\right]=-\kappa \sum_{m} \alpha_{m}^{2} R_{m}(r) Q(t) .
$$

Equation (9) should be averaged over $r$ to be consistent, so

$$
\frac{d}{d t}\left[\sum_{m} \frac{4}{a^{2} \alpha_{m}^{2}} Q(t)-\frac{\kappa}{k} u(t)\right]=\frac{4 \kappa}{a^{2}} Q(t) .
$$

By combining (8) and (10), we obtain a major result

$$
\frac{d \theta(t)}{d t}=\frac{\kappa}{k} \frac{d u(t)}{d t}-\frac{\kappa}{a^{2}} \theta(t)\left[\sum_{m} \frac{1}{a^{2} \alpha_{m}^{2}}\right]^{-1} .
$$

Equation (11) consists of two terms. The first one is due to collisional processes, and the second one is due to thermal conduction.

Bauer [3] gives an expression for the rate of the temperature change in a gas experiencing multiple relaxation processes with uniform axial excitation and no thermal conduction as

$$
\frac{d T}{d t}=\frac{-\boldsymbol{u} \dot{n}_{\text {coll }}}{n C_{v}^{\infty}}
$$

where $\dot{\boldsymbol{n}}_{\text {coll }}=-P \boldsymbol{v} k \overline{\boldsymbol{v}}\left[\boldsymbol{x}^{-1} \boldsymbol{n}-\left(\boldsymbol{n} / R T^{2}\right) \boldsymbol{u}\left(T-T^{0}\right)\right] ; \boldsymbol{u}$ is vector containing the energies of vibrational states, $v$ is a matrix of stoichiometric coefficients for the energy transfer reaction; $\boldsymbol{k}$ is a matrix of energy transfer-rate coefficients; $\boldsymbol{x}$ is a diagonal matrix containing mole fractions of various energy levels; $C_{u}$ is the molar heat capacity of translation and all degrees-of-freedom, that share their energy instantly with translation; $n$ is the number of moles; $R$ is the gas constant; $P$ is the pressure; and $T$ is the absolute temperature.

Equation (12) is substituted into (11), and $\kappa$ in the second term is replaced by $k R T / P C_{v}$ to obtain

$$
\begin{aligned}
\frac{d \theta}{d t}= & \frac{1}{n C_{v}^{\infty}} p v k \bar{v}\left[\boldsymbol{x}^{-1} n-\left(\frac{n}{R T^{2}}\right) \boldsymbol{u}\left(T-T^{0}\right)\right] \\
& -\frac{k R T}{P C_{v}^{\infty}} \frac{\theta(t)}{a^{2}}\left[\sum_{m} \frac{1}{a^{2} \alpha_{m}^{2}}\right]^{-1} .
\end{aligned}
$$

It is clear from (13) that at high pressure the thermal conduction term can be neglected and the rate of change of temperature is mainly due to collisional processes. At low pressure, thermal conduction cannot be neglected and (13) must be solved numerically. The pressure at which the two terms are comparable is determined primarily by the relaxation times of the vibrational modes. For $\mathrm{SF}_{6}$, this pressure is about 200 mtorr.

\section{Spontaneous Emission}

Bauer neglected the effects of spontaneous emission. Spontaneous emission has two experimentally observable effects. At high pressure, since the effect of thermal conduction decreases with increasing pressure, spontaneous emission is a mechanism for transferring energy supplied to the gas by the laser to the cell walls. This effect will parallel thermal conduction and will be important for high gas pressures and long times. At low pressure, spontaneous emission will offer a parallel relaxation path to the slow collisional processes. As a result, the observed relaxation times will be a combination of collisional, spontaneous emission, and wall deexcitation. 
If we assume that the rate of change of occupation number due to absorption and stimulated emission (using matrix notation) is $\dot{n}_{i}$ and the rate of change of occupation number due to spontaneous emission is $\dot{\boldsymbol{n}}_{s}=-\boldsymbol{A n}$ where $\boldsymbol{A}$ is the inverse of the radiative lifetime, then the rate of change of occupation number due to optical transitions is

$$
\dot{\boldsymbol{n}}_{\text {opt }}=-\boldsymbol{A} \boldsymbol{n}+\dot{\boldsymbol{n}}_{\boldsymbol{i}} \text {. }
$$

The total rate of change of occupation number is now

$$
\dot{n}=\dot{\boldsymbol{n}}_{\text {opt }}+\dot{\boldsymbol{n}}_{\text {coll }} \text {. }
$$

Inserting (15) into (16) yields

$$
\dot{\boldsymbol{n}}-\dot{\boldsymbol{n}}_{i}=\dot{\boldsymbol{n}}_{\text {coll }}-\boldsymbol{A n} \text {. }
$$

Equation (16) can be solved numerically. At time $t=$ $0, \boldsymbol{n}$ is set equal to the perturbation in population numbers caused by the laser pulse $\left(\int_{-\infty}^{+\infty} \dot{n}_{\text {opt }} d t\right)$. At later times, $\dot{\boldsymbol{n}}_{\text {opt }}$, the optical absorption term is ignored. Also, at $t=0$ the difference in temperature $T-T^{0}$ is set equal to zero. With these initial conditions, $\dot{\boldsymbol{n}}_{\text {coll }}$ can be determined and then $\dot{n}$ can be computed from (16). At a later time, $t=t+\Delta t$ a new value for $\boldsymbol{n}=\boldsymbol{n}+\dot{n} \Delta t$ is computed. Also a new value for $T-T^{0}$ is determined by using (12). These new values for $T-T^{0}$ and $\boldsymbol{n}$ are used in (9) to compute a new value for $\dot{\boldsymbol{n}}_{\text {coll }}$. Equation (16) is then used to determine a new value for $\dot{n}$. By repeating this procedure again and again for small time changes, the entire temperature evolution can be computed. The pressure change is then computed from the temperature change using the ideal gas law (constant volume is assumed).

\section{Acoustic Effect}

As the incoming radiation propagates down the axis of the tube, absorption of radiation by the gas decreases the intensity in accordance with Beer's Law, $I=I_{o} e^{-\mu z}$. Since the absorbed energy in any small section of the tube differs from that in the next section, the resulting temperature and pressure rise will decrease down the tube axis. A pressure gradient down the tube will, of course, give rise to a net force causing gas near the entrance window to expand into the lower pressure regions further from the entrance window. This expansion is observed as a longitudinally propagating acoustic wave (superimposed upon a uniform pressure rise). For the acoustic wave, the effect of the boundaries is important. Boundaries cause reflections which must be added to the wave developed by the source to give the total pressure. To determine the total pressure at any point, we will use a Green's function approach and the method of images.

According to Morse and Feshbach [9] the solution to the one-dimensional scalar wave equation is

$$
p(x, t)=\int_{0}^{t} d t_{0} \int_{0}^{x} d x_{0} G\left(x, t ; x_{0}, t_{0}\right) p\left(x_{0}, t_{0}\right)
$$

where $x_{0}$ represents the source point; $t_{0}$ represents the time when the source is applied; $x$ represents the observation point where the field is measured; $t$ represents a time later

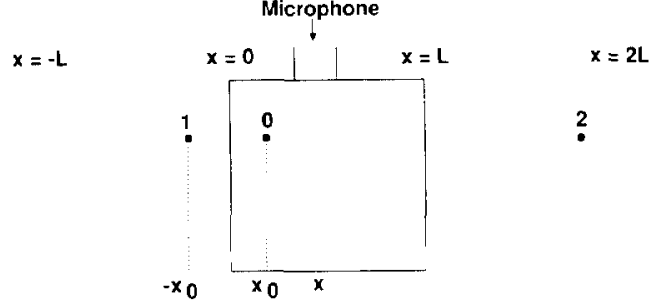

Fig. 2. Sequence of source images. Images are shown at $x=-x_{0}$, and $x$ $=2 L-x_{0}$.

than when the source was applied at $x_{0} ; p(x, t)$ is the pressure at the observation point; $p\left(x_{0}, t_{0}\right)$ is the pressure that describes the source density as a function of space and time; and $G$ is the Green's function describing the effect of the source as it propagates away from $x_{0}$ in the course of time.

To apply the method of images consider the test cell as a cylindrical tube of length $L$ as shown in Fig. 2. The method of images is applied in a stepwise fashion by considering each boundary $x=0$ and $x=L$ in turn. The first boundary encountered is at $x=0$ giving an image at $-x_{0}$; the second is for $x=L$ with an image at $x=2 L-x_{0}$. This process could continue indefinitely, leading to an infinite number of images since there could be an infinite number of reflections. Each image corresponds to one of these reflections.

The number of images actually required will depend on the distance traveled by sound inside the gas sample during the time of interest. For $\mathrm{SF}_{6}$, the sound travels about $40 \mathrm{~cm}$ in the $4 \mathrm{msec}$ typically of interest in our experiments. Since the length of the test cell is $50 \mathrm{~cm}$, this implies that only one image at $x=-x_{0}$ is needed.

The one-dimensional Green's function for our problem is given by

$$
G\left(x, t, x_{0}, t_{0}\right)=2 C \pi\left[1-U\left(\frac{\left|x-x_{0}\right|}{C}-\left(t-t_{0}\right)\right)\right]
$$

where

$$
\begin{aligned}
& U\left[\frac{x-x_{0}}{C}-\left(t-t_{0}\right)\right] \\
& \quad= \begin{cases}1, & \text { when } \frac{\left|x-x_{0}\right|}{C}>\left(t-t_{0}\right) \\
0, & \text { when } \frac{\left|x-x_{0}\right|}{C}<\left(t-t_{0}\right) .\end{cases}
\end{aligned}
$$

These conditions for $U$ give an upper limit for $x_{0} \leq x+$ $C\left(t-t_{0}\right)$ and a lower limit for $x_{0} \geq C\left(t-t_{0}\right)$. For times less than $t=x / C$ there is no need for the image so

$$
p(x, t)=2 \pi C \int_{0}^{t<x / C} \int_{x_{0}=x-C\left(t-t_{0}\right)}^{x_{0}=x+C\left(t-t_{0}\right)} d x_{0} p\left(x_{0}, t_{0}\right) .
$$

After a time $t=x / C$ up to $t=(L-x) / C$, there is one image to consider located at $-x_{0}$ so 


$$
\begin{aligned}
p(x, t)= & 2 C \int_{0}^{t=x / C} d t_{0} \int_{x_{0}=x-C(t-t)}^{x_{0}=x+C\left(t-t_{0}\right)} d x_{0} p\left(x_{0}, t_{0}\right) \\
& +2 \pi C \int_{t=x / C}^{t} d t_{0} \int_{x_{0}=0}^{x_{0}=x+C\left(t-t_{0}\right)} d x_{0} p\left(x_{0}, t_{0}\right) \\
& +2 \pi C \int_{t=x / C}^{t} d t_{0} \int_{x_{0}=x-C\left(t-t_{0}\right)}^{0} d x_{0} p\left(x_{0}, t_{0}\right) .
\end{aligned}
$$

We will assume $p\left(x_{0}, t_{0}\right)$ is directly proportional to $T\left(x_{0}\right.$, $\left.t_{0}\right)$ and solve for $p(x, t)$ only as a final value. The temperature difference $T(x, t)$ at some source point $x_{0}$ is given by $T\left(x_{0}, t_{0}\right)=e^{-\mu x} T\left(t_{0}\right)$, where $\mu$ is the absorption coefficient for the gas. The standard attenuation mechanisms caused by viscosity, thermal conductivity, mass diffusion, and vibrational and rotational relaxation are accounted for by adding a frequency dependent attenuation coefficient. The attenuation coefficient was determined by using a computer program developed by Shields which is described in [9].

$T\left(t_{0}\right)$ was determined by the following steps.

1) Solve (13) numerically to obtain $T(t)=\theta(t)$.

2) Fourier transform $T(t)$ to get $T(\omega)$.

3) Compute the attenuation coefficient $\alpha(\omega)$ as indicated earlier for all frequencies of interest.

4) Compute $\tilde{T}_{t 0}(\omega)=T(\omega) 10^{-x \alpha(\omega) / 20}$.

5) Compute the Fourier transform of $\tilde{T}_{t 0}(\omega)$ to get $T\left(t_{0}\right)$; then substitute this value of $T\left(t_{0}\right)$ into $(21)$ and solve for $p(x, t)$.

\section{Experimental Results}

A typical pressure waveform obtained in this experiment is shown in Fig. 3. This figure shows the variation of pressure amplitude (in arbitrary units) versus time. Energy deposited in the gas by a short laser burst appears first as a small perturbation in the population of vibrationally excited states. Following a sufficient number of collisions, the excess energy will redistribute over internal and translational modes. Changes in temperature and pressure will result as the energy cascades down through the manifold of vibrational states. Fig. 3 shows that the pressure decreases (positive pressure is down) for very short times. Since there is a net gain of energy by the gas, on a longer time scale, the pressure will go positive. For even longer times, the pressure will depend on acoustic effects, spontaneous emission, and thermal conduction to the cell walls, which take the system back to equilibrium.

As the gas pressure is decreased, the amplitude of the pressure wave becomes smaller down to 200 mtorr. At this point, the sign of the pressure change reverses (becomes negative) and the amplitude begins to increase as the pressure is decreased further as shown in Fig. 4.

\section{INTERPRETATION AND CONCLUSION}

The $\mathrm{SF}_{6}$ molecule has 15 vibrational degrees-of-freedom and six fundamental modes [10]. It strongly absorbs

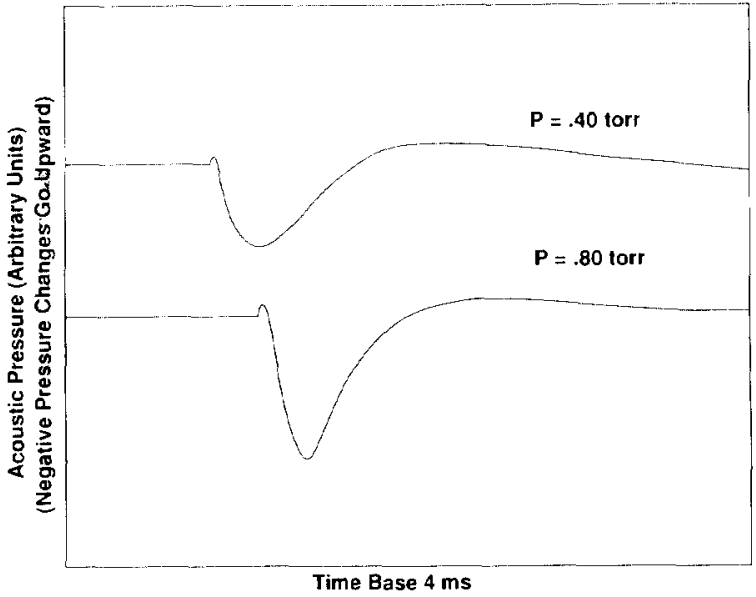

Fig. 3. Typical pressure waveform (positive pressure is down).

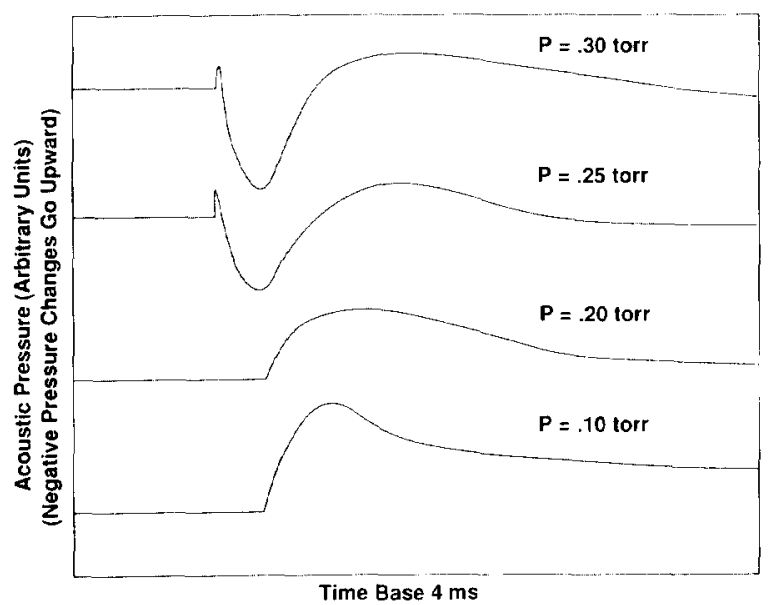

Fig. 4. Change in sign of pressure amplitude as the gas pressure is decreased (positive pressure is down).

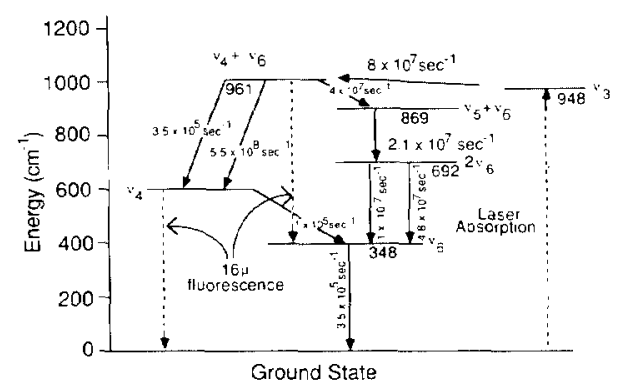

Fig. 5. Partial vibrational energy level diagram for $\mathrm{SF}_{6}$. Numbers along solid lines give rate constants assumed in the model. Numbers below levels give the energy in $\mathrm{cm}^{\prime}$.

$10.6 \mu \mathrm{m} \mathrm{CO} 2$ laser radiation [11] because most of the absorbing molecules are in the ground or lowest excited vibrational state. To interpret the experimental data of Figs. 3 and 4 first consider the energy level diagram of $\mathrm{SF}_{6}$. Fig. 5 shows a few of the low-lying levels [12].

Lifetimes for spontaneous emission can be deduced from a variety of experimental and theoretical work. The radiative lifetime of the $\nu_{3}$ mode was taken to be $8 \mathrm{~ms}$ [12]. Using the results of [13], the radiative lifetime for the $\nu_{4} \rightarrow 0$ process was taken to be 17 times the $\nu_{3}$ lifetime 
or $140 \mathrm{~ms}$. Finally, based upon the results of [14], the lifetime for the $\nu_{4}+\nu_{6} \rightarrow \nu_{6}$ process was taken to be 1.6 times that for $\nu_{4} \rightarrow 0$ or $225 \mathrm{~ms}$.

The first experimental observation to be considered is the decrease in gas temperature and pressure immediately following the input of energy from the laser. This seems to violate laws of thermodynamics. To understand this effect, examine the result of competing energy transfer processes which take place in the gas. Ignore, for now, effects of thermal conduction to walls and spontaneous emission.

The laser excites the $\nu_{3}$ mode which becomes over populated. From Fig. 5 it can be seen that the easiest way for the excited molecules at level $\nu_{3}$ to reach equilibrium is to undergo a $v-v$ transfer to a slightly higher energy level $\left(\nu_{4}+\nu_{6}\right)$. The energy deficit in this reaction must come from somewhere. It comes from translation; the gas cools. As time progresses, energy in the upper vibrational levels cascades downwards returning energy to translation resulting in heating.

Now consider what will happen when the pressure is so low that thermal conduction and spontaneous emission are faster than the transfer of energy to translation but slower than the initial $v-v$ processes, which causes the gas to cool. The result, shown in Fig. 4, is that the gas initially cools but rather than transferring excess energy to translation, it is transferred to the tube walls. Since the test cell has a large specific heat, one observes only cooling of the gas.

There are two major points to be made concerning this experimental observation. First, the very fact that the initial pressure change is negative demonstrates that the initial energy transfer step must be endothermic $(\Delta E<0)$. Second, for the first time net cooling of a gas following energy deposition has been observed. The practical consequences of this observation have not yet been exploited.

Referring back to Fig. 3, the second experimental observation is that in the intermediate pressure regime, the curve does not return to zero after decaying; it overshoots below equilibrium. This is due to acoustic effects, which cause the curve to go below equilibrium, returning slowly to equilibrium on a longer time scale.

It can be seen from Fig. 3, also, that the time from the peak of the curve to the point where it goes below zero is $0.40 \mathrm{~ms}$ for a pressure of 0.4 torr and $0.48 \mathrm{msec}$ for a pressure of 0.80 torr. Another feature of this figure is that the ratio between the negative part of the curve to the positive part is $\frac{1}{5}$ and $\frac{1}{4}$ for pressures of 0.8 and 0.4 torr, respectively. By assuming an effective test cell radius of $0.06 \mathrm{~cm}$, we were able to fit the computed curves to experimental data quite well as shown in Fig. 6. An effective radius is required because the flat microphone intersects part of the cylindrical cell (radius $2 \mathrm{~mm}$ ). Fig. 7 compares experimental data with computed points for the low pressure regime ( 0.1 torr). From Figs. 6 and 7 , it can be seen that the computed curves agree well with the experimental observations.

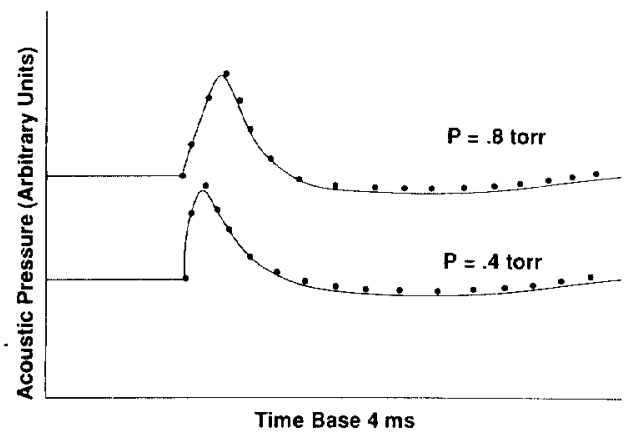

Fig. 6. Fitting of experimental data with computed points. The solid line denotes experimental data, and the dots show computed points. Positive pressure is up.

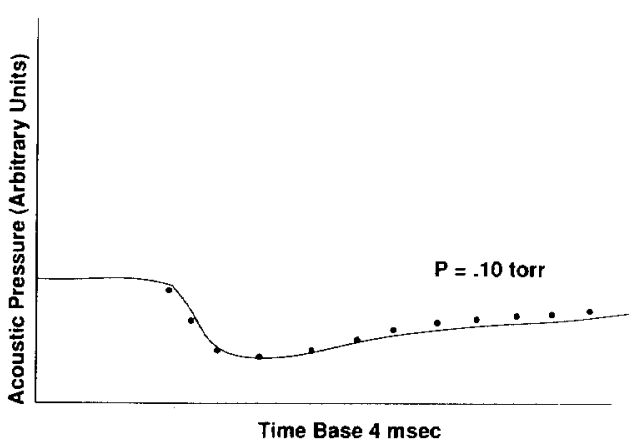

Fig. 7. Fitting of experimental data with computed curve (low pressure regime). The solid line denotes experimental data, and the dots show computed points

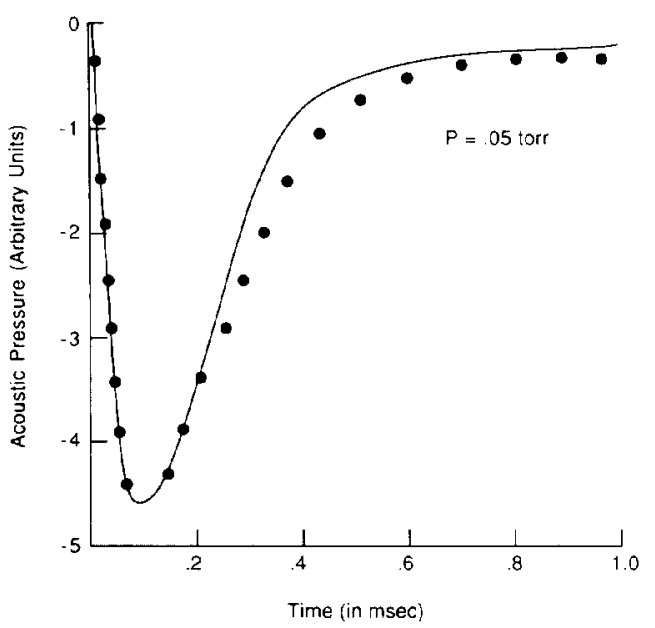

Fig. 8. Effect of spontaneous emission at low pressure. The solid line denotes effect with spontaneous emission, and the dots show effect without spontaneous emission.

Fig. 8 shows two computed curves at low pressure $(0.05$ torr). The solid curve shows the pressure wave when spontaneous emission is included. The solid points show the pressure wave when spontaneous emission is excluded. It can be seen from this figure that the dashed curve has the more rapid decay time; spontaneous emission offers a parallel pathway for deexcitation processes at low pressures.

No experimental technique can completely map the transfer of energy between the various modes in complex 
TABLE I

Energy Transfer Reaction Scheme

\begin{tabular}{crr}
\hline \hline & \multicolumn{1}{c}{ Reaction' } & \multicolumn{1}{c}{$\begin{array}{c}\text { Rate } \\
\left(\mathrm{s}^{-1} \mathrm{~atm}^{-1}\right)\end{array}$} \\
\hline 1 & $\mathrm{SF}_{6}\left(\nu_{3}^{*}\right)+\mathrm{SF}_{6} \rightleftarrows \mathrm{SF}_{6}\left(\nu_{4}^{*}+\nu_{6}^{*}\right)+\mathrm{SF}_{6}$ & $8 \times 10^{7}$ \\
2 & $\mathrm{SF}_{6}\left(\nu_{4}^{*}+\nu_{6}^{*}\right)+\mathrm{SF}_{6} \rightleftarrows \mathrm{SF}_{6}\left(\nu_{4}^{*}\right)+\mathrm{SF}_{6}\left(\nu_{6}^{*}\right)$ & $5.5 \times 10^{8}$ \\
3 & $\mathrm{SF}_{6}\left(\nu_{4}^{*}\right)+\mathrm{SF}_{6} \rightleftarrows \mathrm{SF}_{6}\left(\nu_{6}^{* *}\right)+\mathrm{SF}_{6}$ & $1 \times 10^{5}$ \\
4 & $\mathrm{SF}_{6}\left(\nu_{6}^{* *}\right)+\mathrm{SF}_{6} \rightleftarrows \mathrm{SF}_{6}\left(\nu_{6}^{*}\right)+\mathrm{SF}_{6}\left(\nu_{6}^{*}\right)$ & $1 \times 10^{7}$ \\
5 & $\mathrm{SF}_{6}\left(\nu_{6}^{* *}\right)+\mathrm{SF}_{6} \rightleftarrows \mathrm{SF}_{6}\left(\nu_{6}^{*}\right)+\mathrm{SF}_{6}$ & $4.8 \times 10^{6}$ \\
6 & $\mathrm{SF}_{6}\left(\nu_{6}^{*}\right)+\mathrm{SF}_{6} \rightleftarrows \mathrm{SF}_{6}+\mathrm{SF}_{6}$ & $3.5 \times 10^{5}$ \\
7 & $\mathrm{SF}_{6}\left(\nu_{4}^{*}+\nu_{6}^{*}\right)+\mathrm{SF}_{6} \rightleftarrows \mathrm{SF}_{6}\left(\nu_{4}^{*}\right)+\mathrm{SF}_{6}$ & $3.5 \times 10^{5}$ \\
8 & $\mathrm{SF}_{6}\left(\nu_{4}^{*}+\nu_{6}^{*}\right)+\mathrm{SF}_{6} \rightleftarrows \mathrm{SF}_{6}\left(\nu_{5}^{*}+\nu_{6}^{*}\right)+\mathrm{SF}_{6}$ & $3 \times 10^{7}$ \\
9 & $\mathrm{SF}_{6}\left(\nu_{4}^{*}+\nu_{6}^{*}\right)+\mathrm{SF}_{6} \rightleftarrows \mathrm{SF}_{6}\left(\nu_{6}^{* *}\right)+\mathrm{SF}_{6}$ & $3.1 \times 10^{7}$ \\
\hline
\end{tabular}

'An asterisk indicates one quantum of vibrational energy.

molecules. Each technique is particularly sensitive to different energy transfer reactions. Only by combining results from all experimental sources does a complete picture begin to emerge. The first step in this effort to map the transfer of energy was to devise a relaxation model which would reproduce prior fluorescence [7], [15], double resonance [16], and ultrasonic [2] results using the general theory of Bauer [3]. Then the same relaxation model and rate constants were used to predict theoretical spectrophone response. The computed response was then compared to experimental measurements. This process was repeated until a relaxation scheme agreeing with all data evolved (See Table I). During this process, the effects of spontaneous emission and acoustic propagation were calculated using standard values for radiative lifetimes, speed of sound in $\mathrm{SF}_{6}$, etc. The only adjustable parameters are the multitude of energy transfer rate constants.

Several results of our attempt to fit experimental data are of interest. First, as might be expected, ultrasonic relaxation times are most sensitive to reaction 6 . Since reaction 7 is nearly identical to reaction 6 , it should have the same rate. Reactions 3,8 , and 9 had to be included to yield suitable $16 \mu$ fluorescence decay times. It should be noted that these rates are not unique; different combinations will give satisfactory agreement to laser fluorescence measurements. The resonant reactions, reactions 2 and 4 need only be fast, exact values have only a small effect on fluorescence and other results.

Though the spectrophone measurements reported here provide qualitative information to assist in the formulation of an accurate relaxation scheme, extracting quantative information from the spectrophone data is complicated by the competing processes. The spectrophone measurements are most sensitive to reactions 1 and 6 . The experimental work reported here can only provide improved values for these two reaction rates. The uncertainty (20 percent for reaction 1 and 10 percent for reaction 6) is a result of the fact that different values of other rates in the reaction scheme allow different values of rates from reactions 1 and 2. At very low pressures (less than
300 mtorr) only reaction 1 affects the predictions; the long term evolution is dominated by thermal conduction.

\section{Summary}

This study leads to two conclusions. First, the spectrophone can be a useful tool to distinguish between energy transfer pathways but a complete physical model must rely on complimentary experiments. Second, the combination of molecular energy transfer, thermal conduction, spontaneous emission, and acoustic propagation can lead to a pressure response which is a very complex function of the concentration of the absorbing specie.

\section{ACKNOWLEDGMENT}

The authors gratefully acknowledge early measurements by Rance Fortenberry.

\section{REFERENCES}

[1] A. G. Bell. "Production of sound by radiant energy," Amer. J. Sci. $21,463-490$ (1881).

[2] T. L. Cottrell and J. C. McCoubrey, Molecular Energy Transfer in Gases. London: Butterworths, 1961, pp. 64-73.

[3] H. Bauer, "Son et Lumiere or the opto-acoustic effect in multilevel systems,"'J. Chem. Physi., vol. 57, pp. 3130-3145, 1972.

[4] H. E. Bass and H. X. Yan, "Pulsed spectrophone measurements of vibrational energy transfer in $\mathrm{CO}_{2}$, " J. Acoust. Soc. Amer., vol. 74, no. 6 , pp. $1817-1825,1983$.

[5] R. D. Bates, J. R. George, W. Flynn, and J. T. Knudtson, "Laserinduced 16 fluorescence in $\mathrm{SF}_{6}$ : Acoustic effects," J. Chem. Phys., vol. 53, no. 9, pp. 3621-3631, 1970.

[6] I. N. Sneddon, Fourier Transforms. New York: McGraw-Hill, 1951, p. 202.

[7] R. D. Bates, Jr., J. T. Knudston, and George W. Flynn, "Laser Induced Infrared Fluorescence: Thermal Heating, Mass Diffusion, and Collisional Relaxation in $\mathrm{SF}_{6}, " J$. Chem. Phys. 57, 4174-4190 (1972).

[8] H. S. Carslaw and J. C. Jaeger, Conduction of Heat in Solids. London: Oxford Univ., 1959, p. 198.

[9] P. M. Morse and H. Feshbach, Methods of Theoretical Physics I. New York: McGraw Hill, 1953, pp. 834-845.

[10] F. D. Shields, K. P. Lee, and W. J. Wiley, "Numerical solution for sound velocity and absorption in cylindrical tubes," J. Acoust. Soc. Amer., vol. 37, pp. 724-729, 1965.

[11] G. Herzberg, Infrared and Raman Spectra of Polyatomic Molecules. New York: Van Nostrand, 1945 , p. 337

[12] W. Fuss and J. Hartmann, "IR absorption of $\mathrm{SF}_{6}$ excited up to the disassociation threshold," J. Chem. Phys., vol. 70, p. 5468, 1979.

[13] G. Koren, I. Levin, and U. P. Oppenheim, "Slow intermolecular redistribution of vibrational energy in $\mathrm{SF}_{6}$ gas excited by a TEA $\mathrm{CO}_{2}$ laser," Optics Commun., vol. 32, pp. 257-260, 1980.

[14] W. B. Person and J, Overend, "Infrared intensities: A model for the qualitative prediction of the vibrational strengths of $\mathrm{SF}_{6}$ and $\mathrm{UF}_{6}$," J. Chem. Phys., vol. 66, pp. 1442-1448, 1977.

[15] W. B. Person and K. C. Kim, "Vibrational anharmonicity constants for $\mathrm{SF}_{6}$. I. A $16 \mu$ diode laser study of transitions in the $\nu_{4}$ region," J. Chem. Phys., vol. 69, pp. 2117-2125, 1978.

[16] R. D. Bates, Jr., J. T. Knudtson, G. W. Flynn, and A. M. Ronn, "Energy transfer among excited states of $\mathrm{SF}_{6}$, " Chem. Phys. Lett., vol. 8 , pp. $103-107,1971$

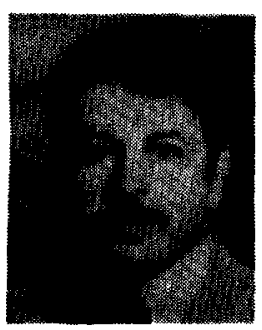

Manaf Hassan Ali was born in Tikrit, Iraq, on January 29, 1946. He obtained the B.Sc. Degree in physics at the University of Baghdad, Iraq in 1969, the M.Sc. Degree in nuclear and elementary particle physics at the University of London, U.K., in 1973, the M.S. Degree in physics at the Virginia Polytechnic Institute and State University, Blacksburg, VA, in 1982, and the Ph.D. Degree in optoacoustics at the University of Mississippi, University, MI, in 1985.

His professional experience includes Labora- 
tory Instructor at the University of Baghdad in 1969-1971; Lecturer at the University of Baghdad in 1973-1975; Assistant for Student Affairs and the University of Baghdad in 1974-1975; Cultural Counselor at the Iraqi Embassy in London in 1975-1978; and Director General at the Iraqi Ministry of Higher Education in 1978-1980. Upon receipt of his Ph.D. degree he obtained a faculty position in the Physics Department, College of Science, University of Baghdad, Iraq. His primary area of interest is concerned with lasers applied to chemical physics. Dr. Ali was a Scholar of Gulbankian Foundation in 1972-1973. He is a member of the Sigma Pi Sigm Physics Honor Society and an Associate Member of the Acoustical Society of America.

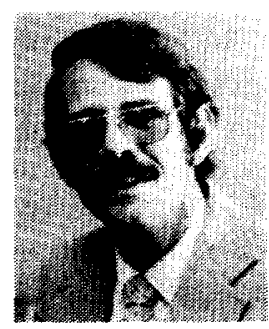

Henry E. Bass was born in Tulsa, OK on October 31, 1943. He received the B.S. degree in physics in 1965 and the Ph.D. degree in physics in 1971 both from Oklahoma State University.

He joined the faculty of the Department of Physics and Astronomy at the University of Mississippi in 1970, where his primary interest has been in physical acoustics. He is also Acting Director of the Acoustics Research and Development Division of the Institute for Technology Development.
Dr. Bass is a fellow of the Acoustical Society of America and Associate Editor of the Journal of the Acoustical Society of America.

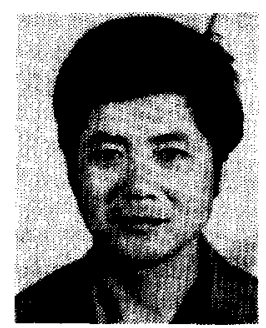

Hai-Xing Yan was bom in Beijing, People's Republic of China, on July 28,1942 . He graduated in 1964 from the Department of Chemical Physics, University of Science and Technology of China (USTC), Beijing, China (now in Hefei, Anhui Province, China).

He was employed by USTC from 1964 to 1973. He then gained a position as Research Scientist in the Division of Laser Research, Institute of Mechanics, Chinese Academy of Sciences, Beijing, China, until 1981. He accepted the position of Visiting Research Scientist at the Physical Acoustics Research Laboratory, The University of Mississippi from 1981-1984. He then returned to the Division of Laser Research where he is presently employed. His primary area of research interest is the numerical simulations and experimental measurements of energy transfer processes in gas by means of lasers and nonequilibrium flow in gas flow lasers and physicochemical gas dynamics.

Dr. Yan is a member of the Chinese Society of Theoretical and Applied Mechanics and the Scientific Advisory Committee of International Symposium on Gas Flow and Chemical Lasers. 